INPLASY

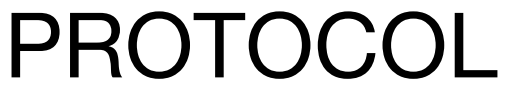

To cite: Yuan et al. The clinical efficacy of Simiao Yong'an Decoction in the treatment of rheumatoid arthritis: A protocol for systematic review and meta analysis. Inplasy protocol

202140069. doi:

10.37766/inplasy2021.4.0069

Received: 12 April 2021

Published: 12 April 2021

Corresponding author:

Shizhong Chen

chenshizhong66@163.com

Author Affiliation:

School of Pharmacy, Peking

University

Support: N0. 2018ZDCXL-

SF-01-05.

Review Stage at time of this

submission: Data extraction.

Conflicts of interest:

None declared.

\section{The clinical efficacy of Simiao Yong'an Decoction in the treatment of rheumatoid arthritis: A protocol for systematic review and meta analysis}

Yuan, MM1; Yin, $\mathrm{MH}^{2}$; Sun, MM³; Chen, SZ4 .

Review question / Objective: Simiao Yong'an Decoction plays an important role in the clinical treatment of rheumatoid arthritis (RA) and has been widely used. Simiao Yong'an Decoction has the effects of clearing away heat, detoxifying, activating blood and dredging collaterals. It is an ancient prescription for treating febrile gangrene. It is believed that Simiao Yong'an Decoction can improve the therapeutic effect of RA. This program aims to systematically evaluate the clinical efficacy of Simiao Yong'an Decoction in the treatment of RA.

Condition being studied: Rheumatoid arthritis. We will search the China National Knowledge Infrastructure (CNKI), Wanfang database, VIPdatabase, the studies in PubMed/MEDLINE, the Cochrane Library, Embase, the Chinese Biomedical Database (CBM) and the Chinese Science Citation Database (CSCD). The retrieval time is from the establishment of the database to April 2021. The search terms are "Simiao Yong'an Decoction", "rheumatoid arthritis", and "Clinical observation", which are carried out by combining subject terms and free words.

INPLASY registration number: This protocol was registered with the International Platform of Registered Systematic Review and Meta-Analysis Protocols (INPLASY) on 12 April 2021 and was last updated on 12 April 2021 (registration number INPLASY202140069).

\section{INTRODUCTION}

Review question / Objective: Simiao Yong'an Decoction plays an important role in the clinical treatment of rheumatoid arthritis (RA) and has been widely used. Simiao Yong'an Decoction has the effects of clearing away heat, detoxifying, activating blood and dredging collaterals. It is an ancient prescription for treating febrile gangrene. It is believed that Simiao Yong'an Decoction can improve the therapeutic effect of RA. This program aims to systematically evaluate the clinical 
efficacy of Simiao Yong'an Decoction in the treatment of RA.

Condition being studied: Rheumatoid arthritis. We will search the China National Knowledge Infrastructure (CNKI), Wanfang database, VIPdatabase, the studies in PubMed/MEDLINE, the Cochrane Library, Embase, the Chinese Biomedical Database (CBM) and the Chinese Science Citation Database (CSCD). The retrieval time is from the establishment of the database to April 2021. The search terms are "Simiao Yong'an Decoction", "rheumatoid arthritis", and "Clinical observation", which are carried out by combining subject terms and free words.

\section{METHODS}

Participant or population: The subjects of the study were patients who were diagnosed with rheumatoid arthritis, regardless of age, gender, or race. The diagnostic criteria were based on the 1987 American College of Rheumatology (ACR) diagnostic criteria for RA.

Intervention: The treatment group was treated with Simiao Yong'an Decoction or Simiao Yong'an Decoction combined with conventional hormone drugs.

Comparator: The control group only used chemical drugs, and the use of drugs and medication methods were not limited.

Study designs to be included: All included studies are described as RCTs.

Eligibility criteria: According to the suggestions of a rheumatologist, we designed the inclusion criteria as follows: (1) According to the 1987 American College of Rheumatology (ACR) diagnostic criteria for RA, patients who received RCT were diagnosed as RA. (2) All the paths mentioned are RCTs, and the research subjects are not limited in age, gender, and race. (3) The experimental group was treated with Simiao Yong'an Decoction or conventional chemical drugs based on Simiao Yong'an Decoction. The control group only used commonly used drugs such as non-steroidal anti-inflammatory drugs (NSAIDs), disease-improving antirheumatic drugs (DMARDs), and glucocorticoids. (4) Outcome indicators: the main outcome indicator is the total effective rate, and the secondary indicators: hand grip strength, morning stiffness, degree of joint swelling, ESR, CRP, RF, incidence of adverse reactions. As long as one indicator is included in the outcome indicators of all included studies, it can be included.

Information sources: The China National Knowledge Infrastructure (CNKI), Wanfang database, VIPdatabase, MEDLINE/PubMed, the Cochrane Library, Embase, the Chinese Biomedical Database (CBM) and the Chinese Science Citation Database (CSCD).

Main outcome(s): The total effective rate (TER) the main clinical feature disappearance rate (DROMCF) the minor symptom disappearance rate (DROMS) ter, dromcf and droms were regarded as binary variables and expressed as odds ratio (OR) and described by $95 \%$ confidence interval $(95 \% \mathrm{Cl})$. The joint swelling, morning stiffness, grip strength, C-reactive protein (CRP), erythrocyte sedimentation rate (ESR), rheumatoid factor (RF) as the effect index, and $95 \% \mathrm{Cl}$ for description. The Q test was used for analysis, combined with I2 to quantitatively determine the degree of heterogeneity. If $P \geq 0.1$ and $12<50 \%$, it is considered that there is no statistical heterogeneity.

Quality assessment / Risk of bias analysis: Two researchers read the literature independently, and screened the literature according to the inclusion and exclusion criteria. Use the Cochrane bias risk assessment tool to evaluate the quality of the included literature, from the aspects of random method implementation, allocation concealment, implementation bias, measurement bias, follow-up bias, reporting bias, and other biases, it gives low bias, uncertain bias, and high bias. Solicit third-party opinions in case of disagreements or inability to judge the literature screening and quality evaluation. 
Strategy of data synthesis: Describing the results of the detailed index, the detailed information concealed by the allocation indicates that the risk of selection bias is low. None of the included studies mentioned blinding and was judged to be a high risk of bias. The experimental information in the study is incomplete, incomplete outcome data, and selective reports cannot judge the risk of bias.

Subgroup analysis: After treatment, the total effective rate of the main clinical features, hand grip strength, morning stiffness time, degree of joint swelling, ESR, CRP, RF, and incidence of adverse reactions were analyzed for statistical heterogeneity among the studies.

Sensitivity analysis: Two researchers independently assessed the quality of the included studies, excluded low-quality studies, and analyzed the same data using different statistical models.

Language: Search without language restrictions.

Country(ies) involved: China.

Keywords: Simiao Yong'an Decoction; Rheumatoid arthritis; Meta-analysis.

Contributions of each author:

Author 1 - Mengmeng Yuan - Collect and select literature drafted the manuscript.

Email: yuanmengmeng514@126.com

Author 2 - Meihua Yin - Performed the data extraction.

Email: y5011434@163.com

Author 3 - Mimi Sun - Check and input data.

Email: 2748058753@qq.com

Author 4 - Shizhong Chen - Designed the study and amended the paper.

Email: chenshizhong66@163.com 